\title{
Axonal Transport Rates In Vivo Are Unaffected by Tau Deletion or Overexpression in Mice
}

\author{
Aidong Yuan, ${ }^{1,2}$ Asok Kumar, ${ }^{1,2}$ Corrinne Peterhoff, ${ }^{1}$ Karen Duff, ${ }^{4}$ and Ralph A. Nixon ${ }^{1,2,3}$ \\ ${ }^{1}$ Center for Dementia Research, Nathan Kline Institute, Orangeburg, New York 10962, 2 Departments of Psychiatry and ${ }^{3}$ Cell Biology, New York University \\ School of Medicine, New York, New York 10016, and ${ }^{4}$ Department of Pathology and Taub Institute, Columbia University, New York, New York 10032
}

\begin{abstract}
Elevated tau expression has been proposed as a possible basis for impaired axonal transport in Alzheimer's disease. To address this hypothesis, we analyzed the movement of pulse radiolabeled proteins in vivo along retinal ganglion cell (RGC) axons of mice that lack tau or overexpress human tau isoforms. Here, we show that the global axonal transport rates of slow and fast transport cargoes in axons are not significantly impaired when tau expression is eliminated or increased. In addition, markers of slow transport (neurofilament light subunit) and fast transport (snap25) do not accumulate in retinas and are distributed normally along optic axons in mice that lack or overexpress tau. Finally, ultrastructural analyses revealed no abnormal accumulations of vesicular organelles or neurofilaments in RGC perikarya or axons in mice overexpressing or lacking tau. These results suggest that tau is not essential for axonal transport and that transport rates in vivo are not significantly affected by substantial fluctuations in tau expression.
\end{abstract}

Key words: tau; Alzheimer's disease; neurofilament; snap25; slow axonal transport; fast axonal transport

\section{Introduction}

Tau is an axonal microtubule-associated protein that stabilizes microtubules, which serve as tracks for the axonal transport of membranous organelles (MOs), vesicles, and protein complexes (Hirokawa and Takemura, 2005). The abnormal somatodendritic accumulation of tau in neurofibrillary tangles is one of the two hallmark pathologies in Alzheimer's disease (AD) and correlates strongly with the extent of neuronal cell loss and the degree of cognitive decline. Tau levels in AD brain may be elevated, but this is still controversial (Khatoon et al., 1992). Although there are no known mutations of tau in $\mathrm{AD}$, tau mutations cause FTDP-17 (frontotemporal dementia with parkinsonism linked to chromosome 17) and are associated with the development of corticobasal degeneration, progressive supranuclear palsy, and Pick's disease (Ballatore et al., 2007). These observations unequivocally establish a pathogenic role of tau in neurodegenerative diseases. Interference with microtubule-dependent axonal transport is one possible mechanism by which altered tau exerts neurotoxicity. High levels of htau40 (the longest human tau isoform) overexpression in neuroblastoma, primary cortical neurons, and retinal ganglion cells (RGCs) have been reported to block the trafficking of MOs and neurofilaments, suggesting that rates of fast and slow transport are impaired (Stamer et al., 2002). The effects of higher or lower levels of tau on axonal transport in

Received March 7, 2007; revised Dec. 31, 2007; accepted Jan. 6, 2008.

This work was supported by National Institute on Aging Grant 5R01AG005604 (R.A.N.) and National Institute of Neurological Disorders and Stroke Grant 5P01NS048447 (K.D.). We thank Takahiro Sasaki, Arthur Saltzman, and Seymour Levine for their technical assistance with immunocytochemistry.

Correspondence should be addressed to Dr. Aidong Yuan, Center for Dementia Research, Nathan Kline Institute, New York University School of Medicine, 140 Old Orangeburg Road, Orangeburg, NY 10962. E-mail: yuan@nki.rfmh.org.

D01:10.1523/JNEUROSCI.5242-07.2008

Copyright $\odot 2008$ Society for Neuroscience $\quad$ 0270-6474/08/281682-06\$15.00/0 vivo in the intact nervous system, however, have not been systematically studied.

In the present study, we directly addressed how modulations in the levels of tau influence transport rates in vivo. By analyzing axonal transport in optic axons from two different lines of mice that either overexpress or lack tau, we found that rates of fast and slow transport are not significantly impaired by modulating tau expression. These findings show that axonal transport is not necessarily dependent on the presence of tau and is not significantly inhibited by moderately elevated levels of tau. These studies also underscore the importance of direct analyses of axonal transport in vivo to evaluate potential pathogenic influences on transport efficiency.

\section{Materials and Methods}

Generation of transgenic animals. Tau overexpressing mice were generated as described previously (Duff et al., 2000) that express a tau transgene derived from a human PAC (P1-derived artificial chromosome), H1 haplotype, known as $8 \mathrm{c}$ mice. Generation of tau knock-out mice that have a targeted disruption of exon one of tau were described previously (Tucker et al., 2001).

Axonal transport studies. RGCs of adult $8 \mathrm{c}$, tau knock-out, and wildtype mice (from 3 to 18 months of age) were radiolabeled by intravitreal injection of [ $\left.{ }^{35} \mathrm{~S}\right]$-methionine as described previously (Yuan et al., 2003). These experimental protocols were approved by York University/Nathan Kline Institute Institutional Animal Care and Use Committee under the guidelines of the Institutional Animal Care and Use Committee of the United States.

Tissue preparation, SDS-PAGE, and immunoblot analysis. Retina or each optic pathway segment pooled from three mice was homogenized in $125 \mu \mathrm{l}$ of cytoskeleton extraction buffer and processed as described previously (Yuan et al., 2003). Antibodies used were polyclonal anti-tau antibody LK recognizing only mouse tau and polyclonal anti-tau antibody JM recognizing both human and mouse tau (Mercken et al., 1995).

Immunocytochemistry. Mice were anesthetized, and retina and optic pathways were processed as described previously (Yuan et al., 2006). 


\section{optic nerve retinal sol retinal pel optic nerve retinal sol retinal pel}

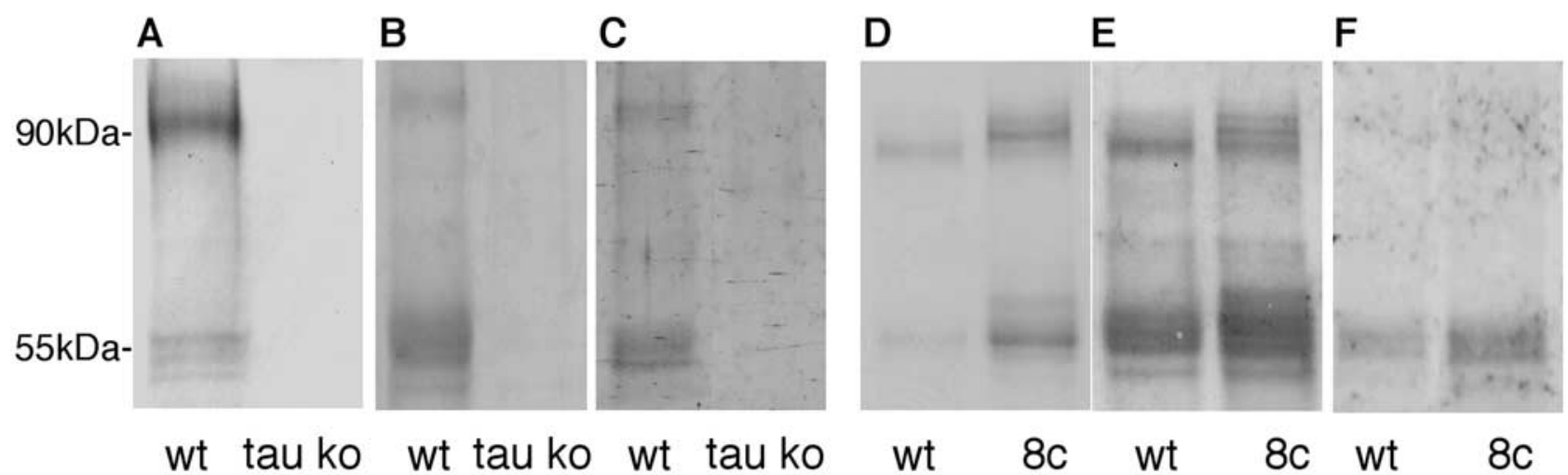

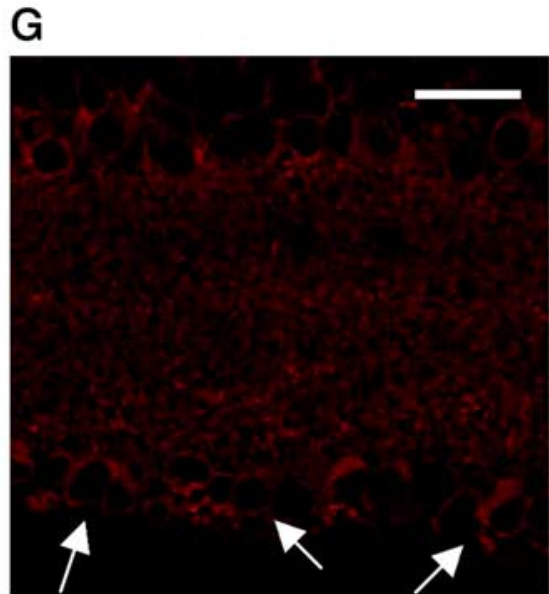

wt
$\mathrm{H}$

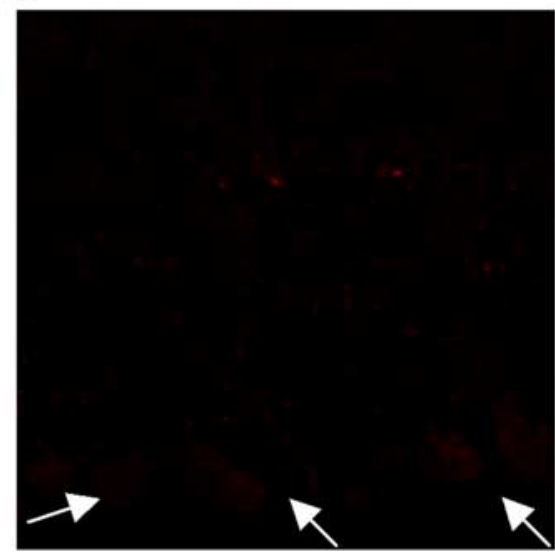

tau ko
I

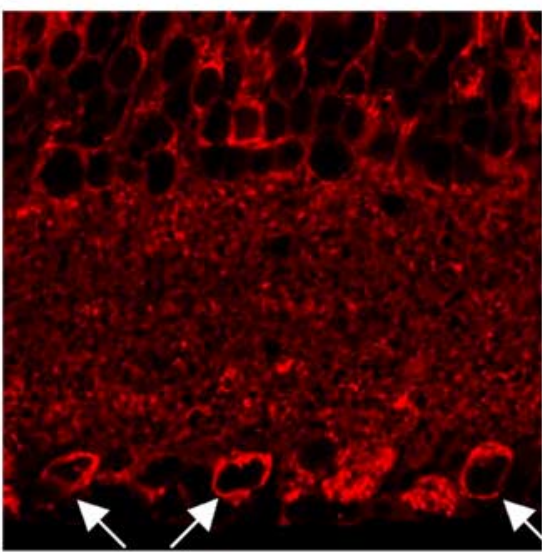

$8 \mathrm{c}$

Figure 1. Tau expression in optic axons and retinas of tau knock-out and overexpressing mice. Total optic nerve extracts $(\boldsymbol{A}, \boldsymbol{D})$ or retinal Triton-soluble $(\boldsymbol{B}, \boldsymbol{E})$ and Triton-insoluble $(\boldsymbol{C}, \boldsymbol{F})$ fractions after separation on $10 \%$ SDS gels were subjected to immunoblot analysis with polyclonal anti-tau antibody. Tau was absent in optic axons $(\boldsymbol{A})$ and retinas $(\boldsymbol{B}, \boldsymbol{C})$ of tau knock-out $(\mathrm{k}$ ) $)$ mice and overexpressed in optic axons $(\boldsymbol{D})$ and retinas $(\boldsymbol{E}, \boldsymbol{F})$ of $8 \mathrm{c}$ mice. Immunofluorescence labeling of tau is absent in retinas from tau knock-out and increased in $8 \mathrm{cmice}(\boldsymbol{G}-\boldsymbol{I})$. RGCs are indicated by white arrows in G-I. Note that the gel loads for wild-type (wt) samples are different, and the blots were developed for different time periods to avoid signal saturation. sol, Soluble; pel, pellet. Scale bar, $20 \mu \mathrm{m}$.

Paraffin-embedded retina was dissected, and 6 - $\mu \mathrm{m}$-thick vibratome sections were processed for immunocytochemistry using total tau antibody Tau A0024 (polyclonal; Dako Cytomation, Carpinteria, CA).

Electron microscopy. The optic nerve and eyes containing the initial $1 \mathrm{~mm}$ of optic nerve were dissected and processed as described previously (Nixon et al., 1994). Quantitation of axonal neurofilaments and measurements of the cross-sectional area of each axon or RGC were performed basically as described previously (Nixon et al., 1994). Visible MOs, including mitochondria, were counted together in RGCs, whereas in axons, mitochondria and other MOs were counted separately. A total of 40 electron micrographs each of retina and optic nerve from two wild-type, two tau knock-out, and two 8c mice were inspected. The average number of MOs $/ \mu \mathrm{m}^{2}$ of cross-sectional area of perikarya were determined in a total of 57 RGC. A total of 10,084 neurofilaments were counted in 428 axon profiles at the $200 \mu \mathrm{m}$ level. Neurofilaments, mitochondria, and other MOs were quantified in a total of 428 optic axons of caliber sizes representing the overall caliber distribution in the optic nerve.

\section{Results}

Tau is absent in optic axons and retinas of tau knock-out mice and overexpressed in those of $8 \mathrm{c}$ mice

Previous studies showed that brain tau is absent in tau knock-out mice (Tucker et al., 2001) and is overexpressed in 8c mice (Duff et al., 2000). To determine whether tau is also absent in RGC in tau knock-out and overexpressed in those of $8 \mathrm{c}$ mice, we performed
SDS-PAGE analyses on optic axons and retinas from those mutant mice and their nontransgenic controls. Optic axons and retinas have been shown to contain several heat-stable tau isoforms in the 50-60 kDa [low molecular weight (LMW) tau] and 90-95 $\mathrm{kDa}$ range [middle molecular weight (MMW) tau] (Mercken et al., 1995). Tau isoforms were identified with antibodies LK and JM. As expected and shown in Figure $1 A-C$, tau knock-out mice lack tau in their optic axons and retinas. In contrast, $8 \mathrm{c}$ mice expressed approximately fourfold more tau in optic axons than did nontransgenic mice (8c, $12766 \pm 863$ vs wt, $3190 \pm 535$; expressed as relative numbers; mean $\pm \mathrm{SD} ; n=4-6$ ) (Fig. $1 D$ ), consistent with previous findings in brain (Duff et al., 2000). The steady-state levels of insoluble ( $8 \mathrm{c}, 4828 \pm 859$ vs wt, $2341 \pm 335$; $n=4)$ and soluble tau ( $8 \mathrm{c}, 8096 \pm 541$ vs wt, $4678 \pm 610 ; n=4)$ in $8 \mathrm{c}$ retinas were approximately twofold higher than the corresponding levels in wild-type mice (Fig. $1 E, F$ ). The relatively low staining of LMW tau in wild-type axons was attributable to the fact that we analyzed total tissue lysates. In our previous study (Mercken et al., 1995), tau was enriched in fractions of Tritonsoluble and heat-stable preparations. The apparent molecular masses of LMW tau species were similar in wild-type and 8c mice, indicating no evidence for changes in the state of phosphorylation of tau in $8 \mathrm{c}$. Consistent with this observation, no significant 
changes were observed using phosphospecific tau antibody AT8 in retinas by quantitative Western blotting and in RGCs by immunofluorescence staining in $8 \mathrm{c}$ mice compared with those in wild-type (data not shown). Differences in the primary structure of human and mouse HMW tau (Kosik et al., 1989) may account for the difference in electrophoretic mobility of these two proteins. Immunofluorescence studies using antibody Tau A0024 revealed higher levels of total tau expression in the RGCs of $8 \mathrm{c}$ (Fig. $1 I$ ) and a complete lack of tau staining in those of tau knock-out mice (Fig. $1 H$ ), consistent with Western blot data.

\section{Axonal transport rates are not} significantly affected by eliminating tau Previous studies revealed loss of brain tau in dysphasic disinhibition dementia 2, a form of frontotemporal dementia (Zhukareva et al., 2001). To determine the effect of tau deletion on slow transport, we analyzed the rates and cargo compositions of slow transport in optic axons of mice lacking tau (Fig. 2) after intravitreal injection of ${ }^{35} \mathrm{~S}$-methionine as described previously (Yuan et al., 2003). At 7 and $14 \mathrm{~d}$ after injection, the optic pathways were cut into $1 \mathrm{~mm}$ segments, which were fractionated into cytoskeleton and soluble fractions with a Triton X-100-containing buffer. Fractionated proteins were separated on 5-15\% SDS-polyacrylamide gels, transferred to nitrocellulose, and visualized by $x$-ray film and phospho-imaging. As shown in Figure 2, the transport rates of slow transport cargoes, neurofilament NF-L, NF-M, and NF-H, were not affected significantly in the absence of mouse tau (Fig. $2 A-D, G-L$ ), possibly reflecting compensatory actions by other microtubule-associated proteins (MAPs) (Harada et al., 1994) and Gem GTPase (Oyama et al., 2004). The calculated peak rates at 7 and $14 \mathrm{~d}$ were $0.21 \mathrm{~mm} / \mathrm{d}(1.5$ $\mathrm{mm}$ divided by $7 \mathrm{~d})$ and $0.18 \mathrm{~mm} / \mathrm{d}(2.5 \mathrm{~mm}$ divided by $14 \mathrm{~d})$ for NF-L, NF-M, and NF-H in both tau-null and wild-type, consistent with previously published results (Yuan et al., 2003, 2006; Yuan, 2006). The peak rate between 7 and $14 \mathrm{~d}$ was calculated as $0.14 \mathrm{~mm} / \mathrm{d}$ ( $1 \mathrm{~mm}$ divided by $7 \mathrm{~d}$ ) for neurofilament proteins in both tau-null and wild-type.

To determine whether fast transport rates are affected by tau deletion, pulse-radiolabeling analyses were performed in tau-null mice. Fast transport was measured in optic axons of tau-null and nontransgenics as described above for slow transport, except the mice in fast transport studies were analyzed at $5 \mathrm{~h}$ after injection. As shown in Figure 2, fast transport rates in tau-null mice were not significantly different from those in wild type. The transport rates of two different fast transport cargoes (represented by p135, one of the most heavily labeled proteins in fast transport with molecular weight of $135 \mathrm{kDa}$ and snap25) quantified by densitometry, were not significantly affected in tau-null mice (Fig. $2 E, F, M, N)$. The calculated peak and front rates for $\mathrm{p} 135$ were 12 and $>38.4 \mathrm{~mm} / \mathrm{d}$, respectively (Fig. $2 M, N$ ), whereas the calculated peak and front rates for soluble snap 25 , a vesicle marker and the most heavily labeled fast transport marker (Hess et al., 1992), were 16.8 and $>38.4 \mathrm{~mm} / \mathrm{d}$, respectively, in both tau-null and wild type. The transport wave peak of snap25 in cytoskeleton fractions moved from the $8 \mathrm{~mm}$ window at $5 \mathrm{~h}$, indicating a peak rate of $>38.4 \mathrm{~mm} / \mathrm{d}$ in both the mutant and wild type (data not shown). These findings indicate that both slow and fast transport rates are not significantly altered in the absence of mouse tau.

\section{Axonal transport rates are not significantly impaired by tau overexpression}

Elevated tau expression has been proposed as a possible basis for impaired axonal transport in AD (Stamer et al., 2002). To determine the effect of moderate tau overexpression on slow transport rate, we analyzed the rates and cargo composition of slow transport in optic axons from $8 \mathrm{c}$ and nontransgenic controls. As shown in Figure 3, the slow transport rates of neurofilament proteins were similar in $8 \mathrm{c}$ and their wild-type controls at $15 \mathrm{~d}$ after injection (Fig. $3 A, B$ ) and at $30 \mathrm{~d}$ postinjection (Fig. $3 C, D$ ). The calculated peak rates at 15 and 30 d were $0.23 \mathrm{~mm} / \mathrm{d}$ ( $3.5 \mathrm{~mm}$ divided 


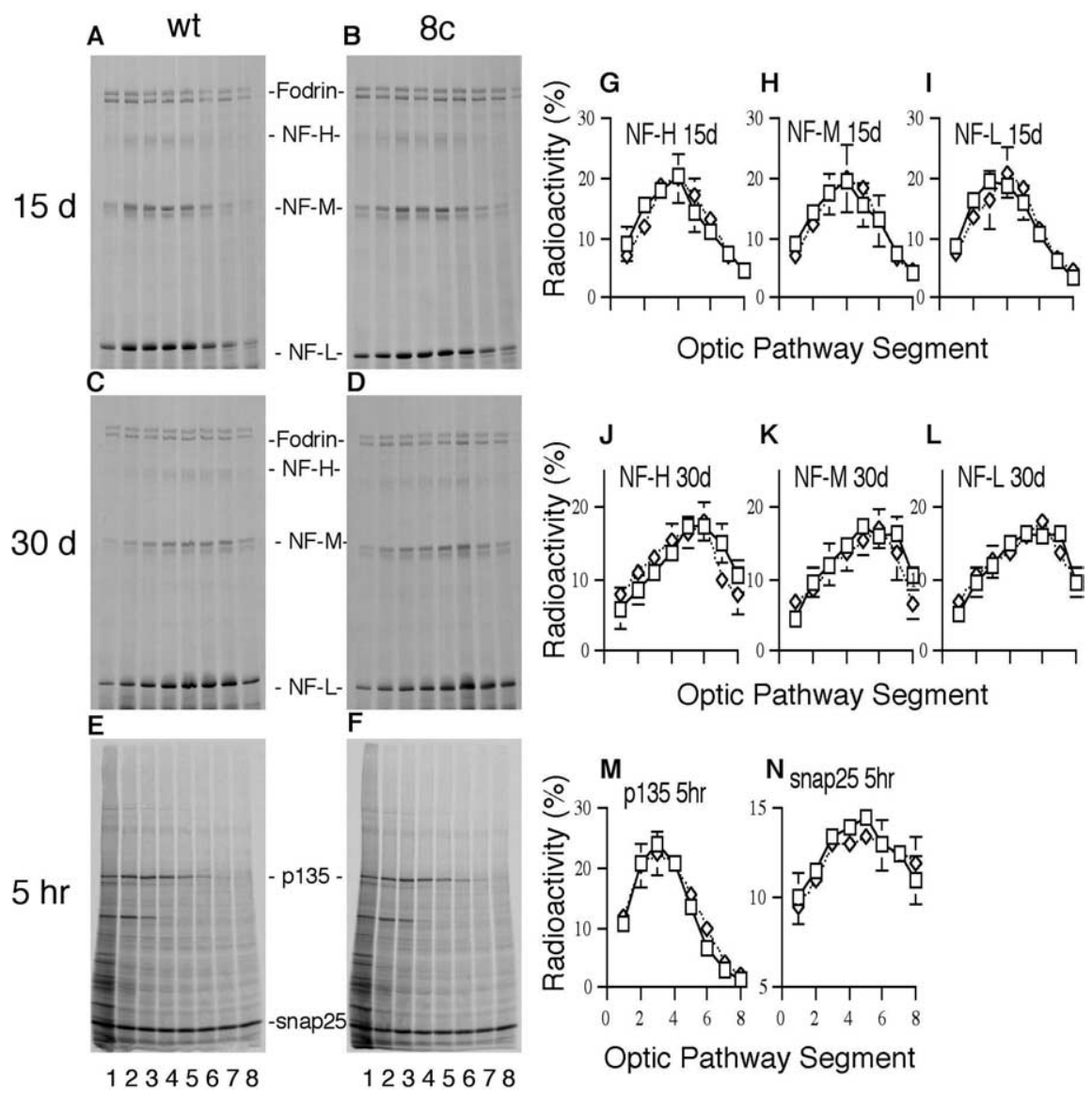

Figure 3. Global transport rates are not significantly impaired by tau overexpression in mice. Axonal transport rates were measured as described in Figure 2, except the postinjection time points were $15 \mathrm{~d}, 30 \mathrm{~d}$, and $5 \mathrm{~h}$. Slow transport rates of neurofilament proteins ( $\boldsymbol{A}-\boldsymbol{D}, \mathbf{G}-\boldsymbol{L} ; 4-7 \%$ SDS-PAGE gels) and fast transport rates of p135 and snap25 $(\boldsymbol{E}, \boldsymbol{F}, \boldsymbol{M}, \boldsymbol{N})$ were not significantly impaired in $8 \mathrm{c}$ mice. Measurements are mean \pm SD. Thirty-six mice ( $188 \mathrm{c}$ mice and 18 wild-type controls) were studied to generate the data, and representative images are shown. Wild-type (wt) and $8 \mathrm{c} \mathrm{mice}$ are represented by squares and diamonds, respectively.

by $15 \mathrm{~d}$ ) and $0.18 \mathrm{~mm} / \mathrm{d}(5.5 \mathrm{~mm}$ divided by $30 \mathrm{~d})$ for NF-L, NF-M, and NF-H in both $8 \mathrm{c}$ and wild type. The peak rate between 15 and $30 \mathrm{~d}$ was calculated as $0.13 \mathrm{~mm} / \mathrm{d}$ ( $2 \mathrm{~mm}$ divided by $15 \mathrm{~d}$ ) for neurofilament proteins in both $8 \mathrm{c}$ and wild type.

To determine the effect of moderate tau overexpression on fast transport, pulse-radiolabeling analyses were performed in $8 \mathrm{c}$ mice. Fast transport was measured in optic axons as described above for tau-null mice, except $8 \mathrm{c}$ mice were used. As shown in Figure 3, $E$ and $F$, the fast transport in $8 \mathrm{c}$ mice was not significantly different from that in wild type. The transport rates of two different fast transport cargoes (represented by protein p135 and snap25) were not significantly impaired in $8 \mathrm{c}$ mice (Fig. $3 M, N$ ). The calculated peak and front rates for p135 were 12 and $>38.4$ $\mathrm{mm} / \mathrm{d}$, respectively, whereas the calculated peak and front rates for snap25 were 16.8 and $>38.4 \mathrm{~mm} / \mathrm{d}$, respectively, in both $8 \mathrm{c}$ and wild-type (Fig. $3 \mathrm{M}, N$ ). These findings indicate that both slow and fast transport rates are not significantly impaired by overexpressing tau in mice.

\section{Normal distributions of NF-L and snap25 after tau deletion or tau overexpression in mice}

Previous in vitro studies indicate that tau overexpression in RGC blocks the transport of vesicles and neurofilaments into axons and causes their accumulation in the cell bodies (Stamer et al., 2002). To determine whether it is also the case in vivo, we per- formed quantitative immunoblotting of retinal proteins with anti-NF-L and snap25 antibodies. As shown in Figure $4 A-D$, no significant alterations in steadystate levels of NF-L and snap 25 were observed in the retinas of tau-null and $8 \mathrm{c}$ mice compared with those of wild type. These results indicate no significant accumulation of neurofilaments and snap25associated vesicles in the retinal ganglion cell bodies in retinas of tau-null or $8 \mathrm{c}$ mice.

Because small, undetectable changes in axonal transport could still lead to abnormal protein distribution over extended periods of time, we performed additional quantitative immunoblot analyses on optic axons to determine the distributions of neurofilaments and snap25-associated vesicles at steady state in tau-null and $8 \mathrm{c}$ mice. The optic pathways were cut into 1 $\mathrm{mm}$ segments, separated into cytoskeleton and soluble fractions, and subjected to 5-15\% SDS-polyacrylamide gels followed by immunoblot analysis using antibodies to NF-L. Quantitative analyses of these immunoblots revealed similar steady-state levels and proximo-distal distributions of NF-L and snap25 along optic axons in taunull, $8 \mathrm{c}$, and wild-type mice (Fig. $3 E-P$ ). Tau distribution pattern was also not significantly affected along optic axons in tau overexpressing mice (data not shown). Lack of organelle and neurofilament accumulations in RGCs and optic axons of taunull and $8 \mathrm{c}$ mice was also confirmed by analyses of the numbers of visible MOs, mitochondria, and neurofilaments from electron micrographs. As shown in Figure $4 Q-T$, the organelle numbers per cross-sectional area $\left(\mu \mathrm{m}^{2}\right)$ of RGC perikaryon and MOs, mitochondria, and neurofilaments per cross-sectional area $\left(\mu \mathrm{m}^{2}\right)$ of optic axons were not significantly altered in tau-null and $8 \mathrm{c}$ mice compared with wild type.

\section{Discussion}

Although the effects of tau expression on axonal transport of neurofilaments and vesicles have been investigated in vitro (Stamer et al., 2002), in vivo effects of tau expression on transport have not been studied systematically. Our studies here on the effects of tau deletion or increased tau expression on axonal transport demonstrate for the first time that general rates of slow and fast transport along optic axons in vivo are not significantly affected by substantial modulations of tau levels. The results of our in vivo studies contrast with earlier proposals that tau overexpression impedes axonal transport, which were based on in vitro studies of neuronal cells showing massive perikaryal accumulations of organelles after high tau overexpression. These studies relied on morphological markers of organelle accumulation rather than direct measurement of axonal transport rates (Stamer et al., 2002). Our results are consistent with a recent report that monomeric tau at levels even 20-fold higher than physiological ones did not affect in vitro axonal transport rates in squid axoplasm (Morfini et al., 2007). At levels 50-fold higher than normal, however, all tau proteins nonspecifically inhibited 
A NFL

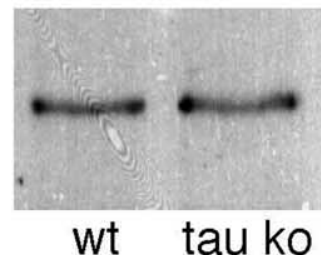

E
B snap25

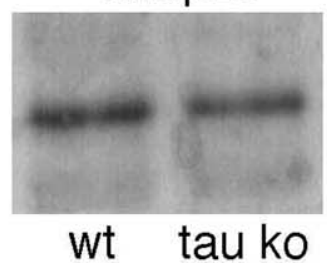

C NFL

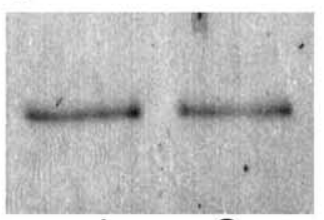

wt $8 c$

I

wt

tau ko G

H

$8 c$
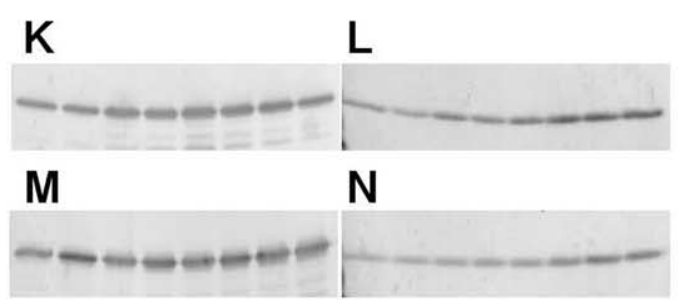

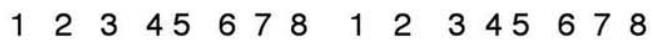

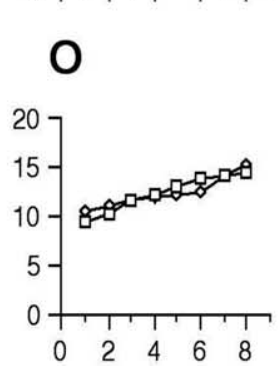

\section{Optic Pathway Segment}
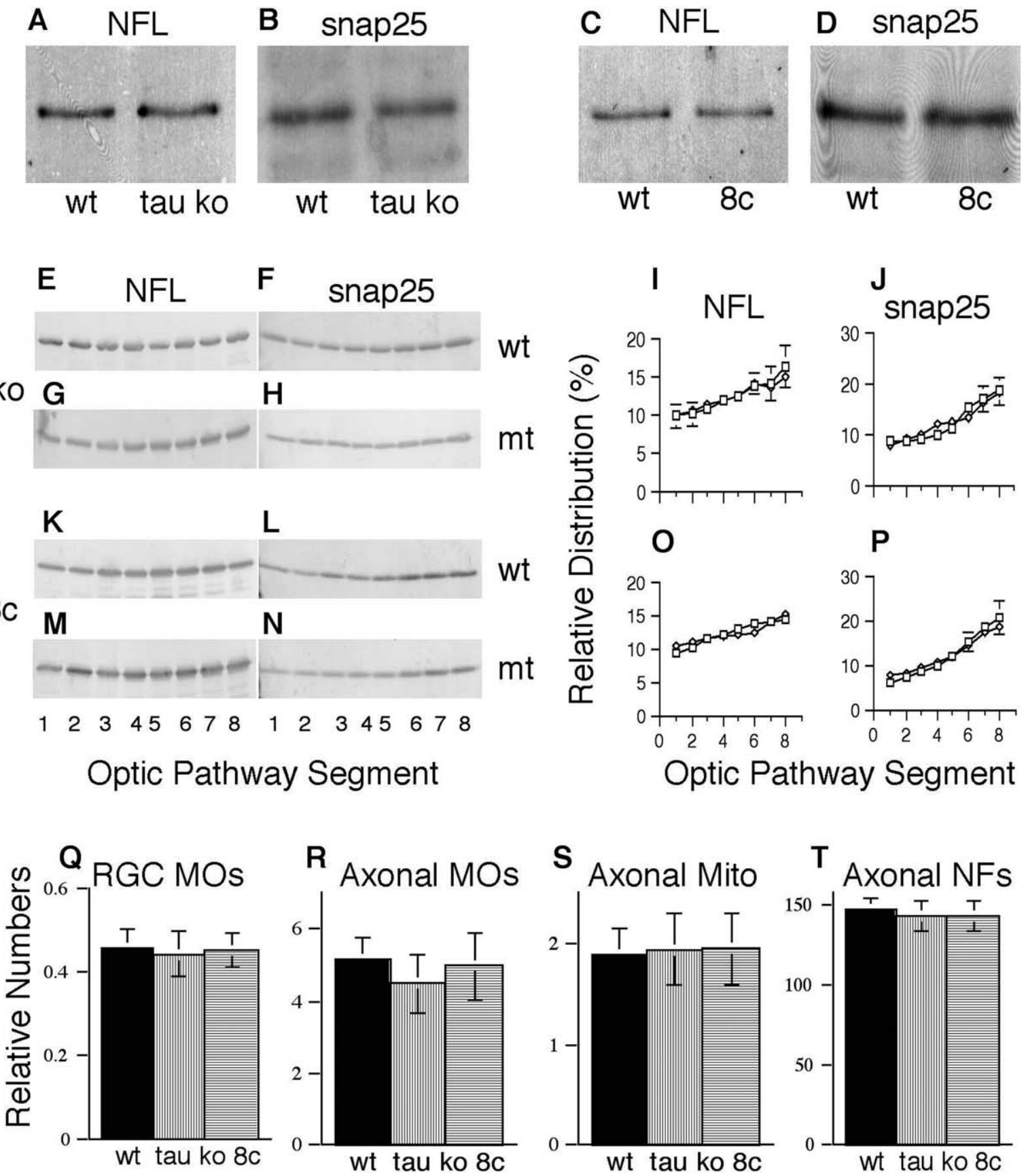

Optic Pathway Segment

Figure 4. Transport markers do not accumulate in retina and distribute normally along optic axons after tau deletion or overexpression in mice. Steady-state levels of NF-L and snap25 in retinas by immunoblot analysis $(\boldsymbol{A}-\boldsymbol{D})$ were not significantly altered, and distributions of these proteins along optic pathways were unchanged $(\boldsymbol{E}-\boldsymbol{P})$ in tau-null and $8 \mathrm{cmice}$ compared with wild-type mice. Measurements are mean \pm SD from 8-12 animals for each group. Wild-type and tau-null or $8 \mathrm{c}$ mice are represented by squares and diamonds, respectively. The numbers of visible M0s counted on electron micrographs expressed as organelle numbers per cross-sectional area $\left(\mu \mathrm{m}^{2}\right)$ of RG( perikaryon in tau-null and $\left.8 \mathrm{c} \mathrm{mice} \mathrm{were} \mathrm{not} \mathrm{significantly} \mathrm{different} \mathrm{from} \mathrm{those} \mathrm{in} \mathrm{wild-type} \mathrm{mice} \mathrm{(} \mathbf{Q}\right)$. Measurements are mean \pm SE from 17-21 RGC for each genotype. Similarly, MOs $(\boldsymbol{R})$, mitochondia $(\boldsymbol{S})$, and neurofilaments $(\boldsymbol{T})$ per cross-sectional area $\left(\mu \mathrm{m}^{2}\right)$ of optic axons were not significantly affected in tau-null and 8c mice compared with wild-type mice. Measurements are mean \pm SE from 119-176 axons of representative caliber for each group. wt, Wild type; Mito, mitochondria; ko, knock-out.

axonal transport, independently of their microtubule-binding activity (Morfini et al., 2007).

The normal transport patterns of NF-L and snap25 along optic axons of tau-null mice indicate that tau is not essential for axonal transport in vivo and may be compensated by other proteins as suggested by the absence of detectable phenotype in mice lacking tau (Harada et al., 1994; Tucker et al., 2001; Oyama et al., 2004). The observation that axonal transport is not hindered by moderate tau expression is also consistent with our ultrastruc- tural data showing that the morphology of RGC and optic axons appears normal, and there is no neuropathology in $8 \mathrm{c}$ mice (Duff et al., 2000). In one study of 12-month-old T44 tau transgenic mice that overexpress the shortest human tau isoform, fast transport rates were slowed in the L5 ventral roots of the spinal cord (Ishihara et al., 1999). However, in a later study by this group, the rate of fast transport in 13-month-old T44 mice exceeded that previously seen in 12-month-old wild-type mice (Zhang et al., 2005). Therefore, no conclusive evidence exists for changes in axonal transport rates after 
tau overexpression in vivo. Our results do not exclude the possibility that tau may interfere with axonal transport when overexpressed at extremely high levels or when its isoform composition or phosphorylation state is altered significantly. The mouse model used here was, in fact, ideal to address the specific issue of tau overexpression effects on transport in the absence of tau hyperphosphorylation or brain pathology.

There are conflicting reports on tau levels in $\mathrm{AD}$ brain (Ksiezak-Reding et al., 1988; Khatoon et al., 1992), and soluble tau levels may actually be reduced $38-65 \%$ in affected brain regions such as frontal and temporal lobes (Ksiezak-Reding et al., 1988). Loss of tau has also been reported to be associated with sporadic and familial tauopathies with frontotemporal dementia (Zhukareva et al., 2001). Our studies of tau deletion and overexpression suggest that the fluctuations of tau steady-state levels that possibly might be achieved in pathological states are unlikely to affect neuronal function by altering global axonal transport rates, in the absence of other changes in tau biophysical properties. Such possibilities have yet to be investigated systematically in animal models in which tau is pathologically altered.

Tau itself is transported at a unique slow rate of transport, which on average is somewhat faster than tubulin and slower than other MAPs (Mercken et al., 1995). The rates of wild-type and mutant tau axonal transport in cultured cortical neurons are similar (Utton et al., 2002). Although the transport of mutant or otherwise modified forms of tau may be specifically affected in pathological situations, such alterations of a subpopulation of tau do not necessarily alter general axonal transport function. In this regard, the overexpression of a NF-H-LacZ fusion protein in transgenic mice inhibits export of a subpopulation of newly synthesized neurofilament protein into axons and induces $\alpha$-internexin and neurofilament triplet to aggregate in neuronal perikarya. However, the transport rate of NF-L that escapes into axons remains normal (Yuan et al., 2006; our unpublished data). Abnormal intraneuronal accumulation of $\alpha$-synuclein in Lewy bodies and Lewy neurites caused by familial Parkinson's disease (FPD)-linked mutations has suggested a defect in axonal transport of $\alpha$-synuclein. However, in transgenic mice overexpressing FPD-linked mutant human $\alpha$-synuclein, the development of Lewy body inclusions in some spinal motor neurons is not associated with alterations in the transport rates of slowly transported proteins, including $\alpha$-synuclein, neurofilaments, and tubulin ( $\mathrm{Li}$ et al., 2004).

In conclusion, our studies directly demonstrate in vivo that the global axonal transport rates are not significantly affected by tau deletion or overexpression in mice. In contrast to reports of perikaryal accumulations of transport cargoes in neuronal cell cultures with high tau overexpression, we find that high levels of tau alone are insufficient to impair general axonal transport function in vivo. Our data do not preclude the possibility that aggregation or dysfunction of pathologically altered tau induces neurodegenerative changes in Alzheimer's diseases that secondarily cause axonal transport dysfunction. These results underscore the value of performing axonal transport analyses in vivo to investigate pathologic consequences of pathogenic proteins on transport of proteins and or organelles.

\section{References}

Ballatore C, Lee VM, Trojanowski JQ (2007) Tau-mediated neurodegeneration in Alzheimer's disease and related disorders. Nat Rev Neurosci 8:663-672.

Duff K, Knight H, Refolo LM, Sanders S, Yu X, Picciano M, Malester B, Hutton M, Adamson J, Goedert M, Burki K, Davies P (2000) Character- ization of pathology in transgenic mice over-expressing human genomic and cDNA tau transgenes. Neurobiol Dis 7:87-98.

Harada A, Oguchi K, Okabe S, Kuno J, Terada S, Ohshima T, Sato-Yoshitake R, Takei Y, Noda T, Hirokawa N (1994) Altered microtubule organization in small-calibre axons of mice lacking tau protein. Nature 369:488-491.

Hess DT, Slater TM, Wilson MC, Skene JH (1992) The $25 \mathrm{kDa}$ synaptosomal-associated protein SNAP-25 is the major methionine-rich polypeptide in rapid axonal transport and a major substrate for palmitoylation in adult CNS. J Neurosci 12:4634-4641.

Hirokawa N, Takemura R (2005) Molecular motors and mechanisms of directional transport in neurons. Nat Rev Neurosci 6:201-214.

Ishihara T, Hong M, Zhang B, Nakagawa Y, Lee MK, Trojanowski JQ, Lee VM (1999) Age-dependent emergence and progression of a tauopathy in transgenic mice overexpressing the shortest human tau isoform. Neuron 24:751-762.

Khatoon S, Grundke-Iqbal I, Iqbal K (1992) Brain levels of microtubuleassociated protein tau are elevated in Alzheimer's disease: a radioimmuno-slot-blot assay for nanograms of the protein. J Neurochem 59:750-753.

Kosik KS, Orecchio LD, Bakalis S, Neve RL (1989) Developmentally regulated expression of specific tau sequences. Neuron 2:1389-1397.

Ksiezak-Reding H, Binder LI, Yen SH (1988) Immunochemical and biochemical characterization of tau proteins in normal and Alzheimer's disease brains with Alz 50 and Tau-1. J Biol Chem 263:7948-7953.

Li W, Hoffman PN, Stirling W, Price DL, Lee MK (2004) Axonal transport of human alpha-synuclein slows with aging but is not affected by familial Parkinson's disease-linked mutations. J Neurochem 88:401-410.

Mercken M, Fischer I, Kosik KS, Nixon RA (1995) Three distinct axonal transport rates for tau, tubulin, and other microtubule-associated proteins: evidence for dynamic interactions of tau with microtubules in vivo. J Neurosci 15:8259-8267.

Morfini G, Pigino G, Mizuno N, Kikkawa M, Brady ST (2007) Tau binding to microtubules does not directly affect microtubule-based vesicle motility. J Neurosci Res 85:2620-2630.

Nixon RA, Paskevich PA, Sihag RK, Thayer CY (1994) Phosphorylation on carboxyl terminus domains of neurofilament proteins in retinal ganglion cell neurons in vivo: influences on regional neurofilament accumulation, interneurofilament spacing, and axon caliber. J Cell Biol 126:1031-1046.

Oyama F, Kotliarova S, Harada A, Ito M, Miyazaki H, Ueyama Y, Hirokawa N, Nukina N, Ihara Y (2004) Gem GTPase and tau: morphological changes induced by gem GTPase in cho cells are antagonized by tau. J Biol Chem 279:27272-27277.

Stamer K, Vogel R, Thies E, Mandelkow E, Mandelkow EM (2002) Tau blocks traffic of organelles, neurofilaments, and APP vesicles in neurons and enhances oxidative stress. J Cell Biol 156:1051-1063.

Tucker KL, Meyer M, Barde YA (2001) Neurotrophins are required for nerve growth during development. Nat Neurosci 4:29-37.

Utton MA, Connell J, Asuni AA, van Slegtenhorst M, Hutton M, de Silva R, Lees AJ, Miller CC, Anderton BH (2002) The slow axonal transport of the microtubule-associated protein tau and the transport rates of different isoforms and mutants in cultured neurons. J Neurosci 22:6394-6400.

Yuan A (2006) Neurofilament protein partnership, export, transport, phosphorylation and neurodegeneration. In: New research on neurofilament proteins (Arlen R, ed), pp 53-79. New York: Nova Science.

Yuan A, Rao MV, Kumar A, Julien JP, Nixon RA (2003) Neurofilament transport in vivo minimally requires hetero-oligomer formation. J Neurosci 23:9452-9458.

Yuan A, Rao MV, Sasaki T, Chen Y, Kumar A, Veeranna, Liem RK, Eyer J, Peterson AC, Julien JP, Nixon RA (2006) $\alpha$-Internexin is structurally and functionally associated with the neurofilament triplet proteins in the mature CNS. J Neurosci 26:10006-10019.

Zhang B, Maiti A, Shively S, Lakhani F, McDonald-Jones G, Bruce J, Lee EB, Xie SX, Joyce S, Li C, Toleikis PM, Lee VM, Trojanowski JQ (2005) Microtubule-binding drugs offset tau sequestration by stabilizing microtubules and reversing fast axonal transport deficits in a tauopathy model. Proc Natl Acad Sci USA 102:227-231.

Zhukareva V, Vogelsberg-Ragaglia V, Van Deerlin VM, Bruce J, Shuck T, Grossman M, Clark CM, Arnold SE, Masliah E, Galasko D, Trojanowski JQ, Lee VM (2001) Loss of brain tau defines novel sporadic and familial tauopathies with frontotemporal dementia. Ann Neurol 49:165-175. 